\title{
ESTADO CIVIL Y SÍNDROME DE BURNOUT EN GERENTES MEDIOS Y SUPERIORES DE LA INDUSTRIA MANUFACTURERA MEXICANA
}

\author{
MARITAL STATUS AND BURNOUT IN MIDDLE AND SENIOR MANAGERS OF THE \\ MEXICAN MANUFACTURING INDUSTRY
}

\author{
Oziely Daniela Armenta Hernández ${ }^{*}$ \\ Aidé Aracely Maldonado Macias ${ }^{* *}$ \\ Manuel Alejandro Barajas Bustillos ${ }^{* * *}$ \\ Arturo Realyvasquez Vargas ${ }^{* * * *}$ \\ Yolanda Angélica Báez López ${ }^{* * * *}$
}

\begin{abstract}
Resumen: El síndrome de burnout es uno de los temas de la salud mental más estudiados a nivel mundial y de creciente interés en el contexto industrial. El presente estudio se llevó a cabo en la industria maquiladora de Ciudad Juárez, México, donde existen 321 industrias manufactureras de exportación que emplean a más de 250.000 personas. Los gerentes medios y superiores están sujetos a fuertes demandas laborales tanto de sus superiores como de sus subordinados y son clave para el logro de los objetivos de la organización, por lo que es importante estudiar los aspectos que afectan su bienestar y salud en el trabajo. Esta población es vulnerable al estrés laboral crónico por lo que se exponen los resultados de la presencia del burnout en una muestra de 932 empleados. Así mismo, se ha reconocido que el estado civil es una variable que puede afectar la aparición de este síndrome. Valores medios se obtuvieron para cada dimensión de burnout por estado civil y se compararon usando ANOVA y la prueba de Tukey con un nivel de confianza del $95 \%$. Se compararon cinco estados civiles distintos. Este estudio encontró algún grado y nivel de agotamiento emocional en todos los estados civiles estudiados. Además, se encontraron diferencias significativas solo en la dimensión de agotamiento emocional, en la cual estar casado es el estado civil que presenta esta diferencia respecto al resto de estados civiles.
\end{abstract}

Palabras clave: Burnout, estado civil, estrés laboral, industria, mandos medios y superiores.

\begin{abstract}
Nowadays, burnout syndrome is one of the most studied mental health topics and has a growing interest in the industrial context. This study was conducted in the manufacturing industry of Ciudad Juarez Mexico. This industry currently employs more than 250,000 individuals. Among them, middle and senior managers are critical job positions. They are subjected to high mental and physical demands from both their superiors and their subordinates. Thus, their work is key to the completion of
\end{abstract}

\footnotetext{
"Universidad Autónoma de Ciudad Juárez. Ciudad Juárez, México. Correo electrónico: al164439@alumnos.uacj.mx. Orcid: https://orcid.org/oooo-0003-1009-5384

${ }^{* *}$ Universidad Autónoma de Ciudad Juárez. Ciudad Juárez, México. Correo electrónico: amaldona@uacj.mx. Orcid: https://orcid.org/oooo-0002-4959-161X. Autor de correspondencia.

${ }^{* * *}$ Universidad Autónoma de Ciudad Juárez. Ciudad Juárez, México. Correo electrónico: al171528@alumnos.uacj.mx. Orcid: https://orcid.org/oooo-ooo3-1155-1847

****Tecnológico Nacional de México/IT Tijuana. Tijuana, México. Correo electrónico: arturo.realyvazquez@tectijuana.edu.mx. Orcid: https://orcid.org/oooo-ooo3-2825-2595

*****Autonomous University of Baja California. Ensenada, México. Correo electrónico: yolanda@uabc.edu.mx. Orcid: https://orcid.org/oooo-ooo1-8418-254X
} 
organizational goals. Managers are vulnerable to chronic work stress, so this study presents results of burnout's presence in a sample of 932 employees. Likewise, it has been recognized that marital status is a variable that can influence the presence of burnout syndrome. Therefore, once mean values were obtained for each burnout dimension by marital status, they were compared using ANOVA and the Tukey's test with a confidence level of $95 \%$. Five marital statuses were compared. As a result, a medium degree of burnout was found for all three dimensions for each marital status studied. This study found that marital status influences burnout's mean scores since ANOVA detects significant differences among the three dimensions. There is evidence of the presence of burnout in all the marital statuses studied. Nevertheless, only the emotional exhaustion dimension presents differences among all the studied marital statuses, where being married statistically differs with the rest of them.

Keywords: Burnout, marital status, work stress, industry, middle and senior managers.

Recepción: 09.04.2021 / Revisión: 12.04.2021 / Aceptación: 04.05.2021

\section{Introducción}

La salud mental de los trabajadores puede ser afectada por diferentes factores. Uno de los más importantes y contagiosos es el estrés, ya que al ver o estar cerca de alguna persona con estrés puede incrementar los niveles de cortisol de acuerdo con el Instituto Mexicano del Seguro Social (Instituto Mexicano del Seguro Social [IMSS], 2018). Actualmente, el estrés laboral es uno de los problemas a los cuales los investigadores están prestando más atención. Esto se debe a que cada vez más empleados son conscientes de su importancia y sus consecuencias. El prevenirlo o tratarlo ayudará a mantener mejores condiciones de salud, tanto física como mental en las organizaciones. Últimamente, el estrés es uno de los desórdenes psicológicos que más afecta la salud de las personas, provocando alteraciones que impactan en su desempeño laboral o el de la empresa (Aguirre et al., 2014; González, 2019). Se define como una reacción psicológica del organismo cuando una persona percibe una situación amenazante, es una respuesta automática y necesaria para la supervivencia. Cuando esta reacción natural ocurre de manera excesiva, se produce una sobrecarga de tensión, la cual provoca efectos adversos en todo el cuerpo. Esto puede provocar alteraciones en la salud e impedir el desarrollo y funcionamiento normal del cuerpo humano (IzquierdoBotica, 2019). Así mismo, el estrés relacionado con el trabajo se describe como un grupo de reacciones físicas y psicológicas cuando los empleados enfrentan demandas ocupacionales que exceden sus conocimientos, habilidades o habilidades. Las reacciones de los trabajadores al estrés incluyen respuestas fisiológicas, emocionales, cognitivas y conductuales. En el modelo de González-Ramírez y Landero-Hernández (2008), el estrés percibido sería el resultado de enfrentar uno o varios factores estresantes y valorar esa situación como estresante y desbordante de los recursos de la persona; la valoración de los recursos incluye la valoración de los recursos propios (autoestima y autoeficacia) y la valoración del apoyo ambiental (apoyo social). Además, variables sociodemográficas, condiciones sociales como organización social, aspectos socioeconómicos, estado civil, rol laboral, género, entre otros, pueden estar involucrados tanto en el origen como en las consecuencias de experiencias estresantes (Sandín, 2003). 
El síndrome de burnout es un tipo de estrés laboral o un estado de agotamiento físico, mental y emocional que puede afectar la autoestima y la salud en general del individuo. Ocurre en dos fases donde, primeramente, hay un lento desequilibrio entre la demanda de trabajo y los recursos para hacer frente a ella. Posteriormente, se presenta un desequilibrio emocional del trabajador y es cuando se produce un cambio de comportamiento hacia el trabajo, haciendo lo mínimo necesario, pero evitando cualquier riesgo. El burnout consta de tres dimensiones: el agotamiento emocional, que es cuando se percibe la experiencia de estar emocionalmente agotado por las exigencias del trabajo; el cinismo, que reconoce el grado que se reconocen actitudes de frialdad y distanciamiento; y, finalmente, la eficacia profesional, que se ocupa de los sentimientos de autoeficacia y realización personal en el trabajo (Guerrero \& Vicente, 2001; Peralta-Ayala \& Moya-Silva, 2017).

Varios estudios han estudiado la relación del burnout con el estado civil de las personas. Uno de estos estudios fue el realizado por Ávila-Toscano et al., (2010) que no encontró diferencia estadística significativa entre ambas variables. También, en el estudio realizado por Picasso-Pozo et al. (2012) no se detectó relación alguna entre el estado civil y el burnout. Por el contrario, Bautista et al. (2013) realizaron un estudio y descubrieron que es más probable que las personas casadas presenten burnout; mientras que el estudio llevado a cabo por Peralta-Ayala y Moya-Silva (2017), afirma que el estar en unión libre favorece el incremento del agotamiento emocional. Además, otro estudio realizado por QuezadaBerumen y González-Ramírez (2011) encontró que el estado civil de las personas se relaciona con los niveles de depresión y estrés en adolescentes embarazadas. Estos estudios dejan en claro que existe una relación directa entre la variable socio demográfica del estado civil de las personas y el burnout.

\section{Objetivos}

Los objetivos de esta investigación son determinar la prevalencia del síndrome de burnout en una muestra de mandos medios y superiores de Ciudad Juárez, México, así como aquellas diferencias significativas entre las dimensiones del burnout por estado civil.

\section{Delimitación}

El presente estudio se realizó en una muestra de mandos medios y altos de la industria maquiladora de Ciudad Juárez, México.

\section{Materiales y métodos}

\section{Metodología}

La muestra para este estudio incluyó a 932 participantes. Para recabar los datos, se utilizó la versión en español del cuestionario de Inventario de Desgaste de Maslach (Maslach Burnout Inventory (MBI), en inglés) desarrollada por (Moreno-Jiménez et al., 2001). El cuestionario incluye 16 preguntas divididas en tres dimensiones que son agotamiento emocional, cinismo 
y eficiencia profesional, y tiene dos secciones. La primera sección mide las dimensiones del burnout y la segunda contiene datos sociodemográficos para describir la muestra. Este último incluye preguntas sobre su estado civil, antigüedad en la empresa, jornada laboral y puesto de trabajo, entre otras.

El estudio descriptivo de burnout se presenta de 3 formas diferentes, mediante puntuaciones medias, grados y niveles de burnout, de acuerdo con la metodología de Guerrero y Vicente (2001). Una vez que los valores medios se obtuvieron para cada dimensión de burnout por estado civil, el tratamiento de los datos se realizó mediante un análisis de varianza (Analysis of Variance, ANOVA) comparando los valores medios de las puntuaciones de burnout para determinar diferencias significativas en la presencia del síndrome entre los estados civiles estudiados. Además, se aplicó la prueba de Tukey con un nivel de confianza del 95\% para comparar las medias individuales entre cinco estados civiles distintos: casado, soltero, divorciado, viudo y en unión libre.

\section{Grados de burnout}

El grado de burnout para cada dimensión se obtiene cuando los valores de las puntuaciones medias se encuentran en el tercio superior, medio o inferior de los posibles valores de rango determinados por los puntos de corte de los percentiles 33 y 66 de la muestra estudiada.

\section{Niveles de burnout}

El nivel de burnout se puede presentar en una nueva variable categórica explicada en 5 categorías. Los niveles se obtienen de las 27 combinaciones derivadas de los tres grados de burnout. Los niveles son: ninguno, bajo, moderado, alto y extremadamente alto.

\section{Resultados y discusión}

A continuación, se presentan los análisis descriptivos de la muestra. La figura 1 presenta la distribución de la muestra por estado civil, en la figura 2 se observa la distribución de la muestra por antigüedad en el trabajo en meses, la figura 3 muestra la distribución de las horas trabajadas, la figura 4 hace referencia a la distribución de la muestra por puesto de trabajo, y en la figura 5 se presenta la muestra distribuida por género. 
Figura 1. Estado civil de los participantes en la muestra (\%).

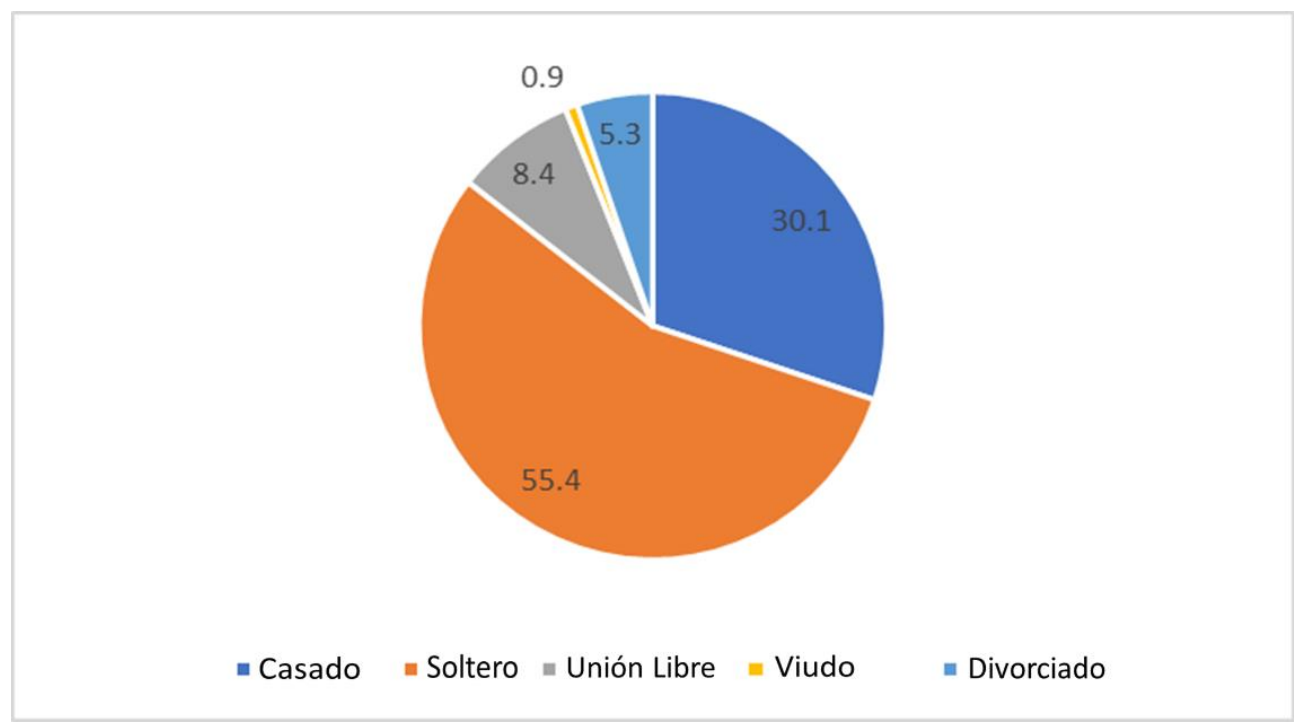

Figura 2. Antigüedad de los participantes, en meses.

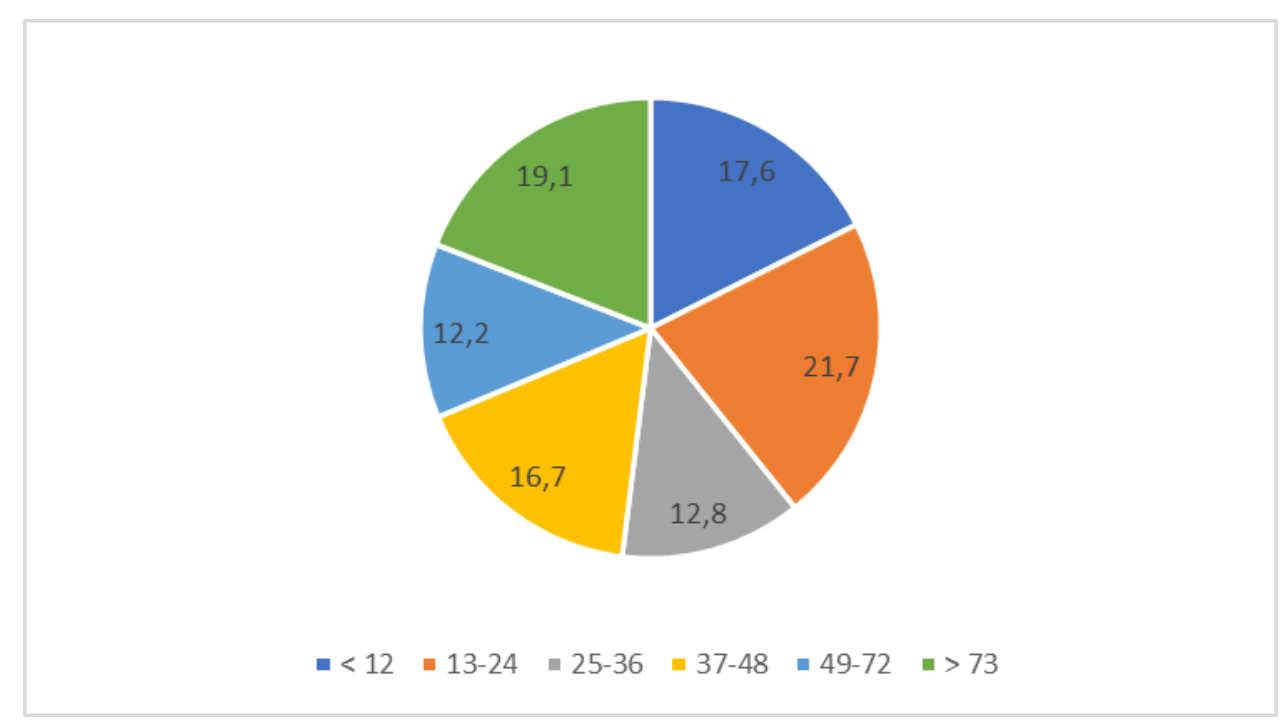


Figura 3. Edad de los participantes (años).

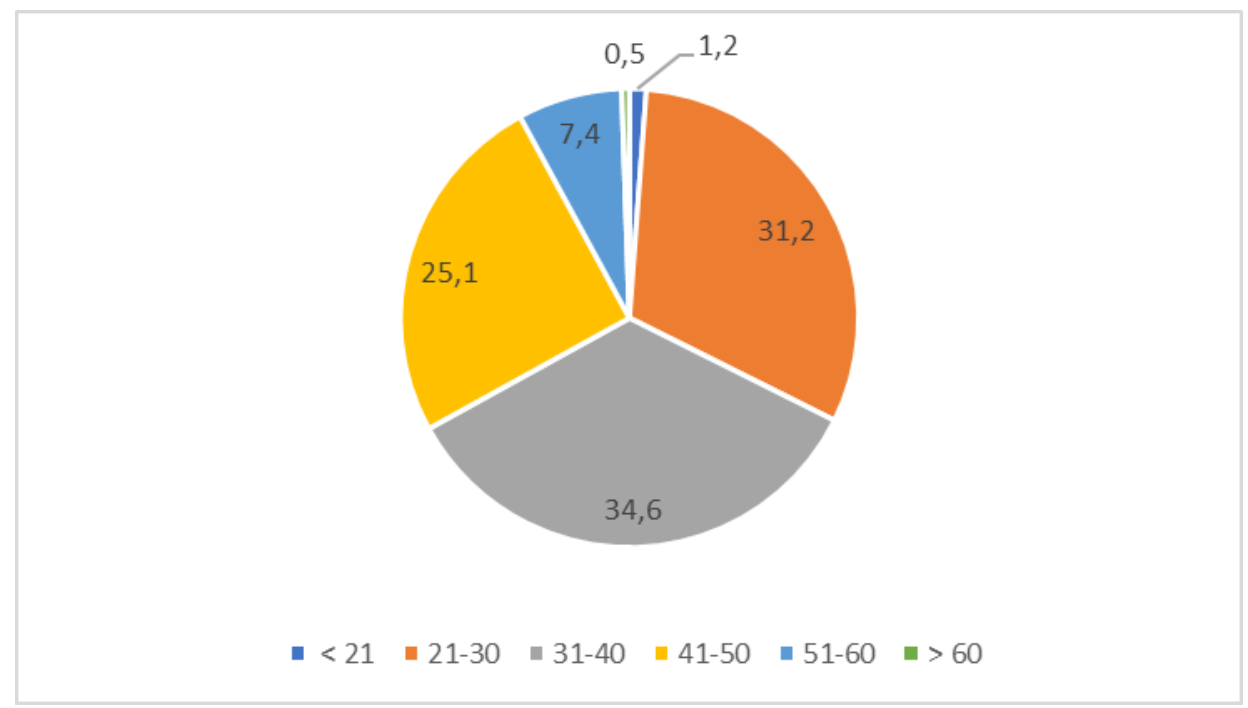

Figura 4. Puestos de trabajo de los participantes (\%).

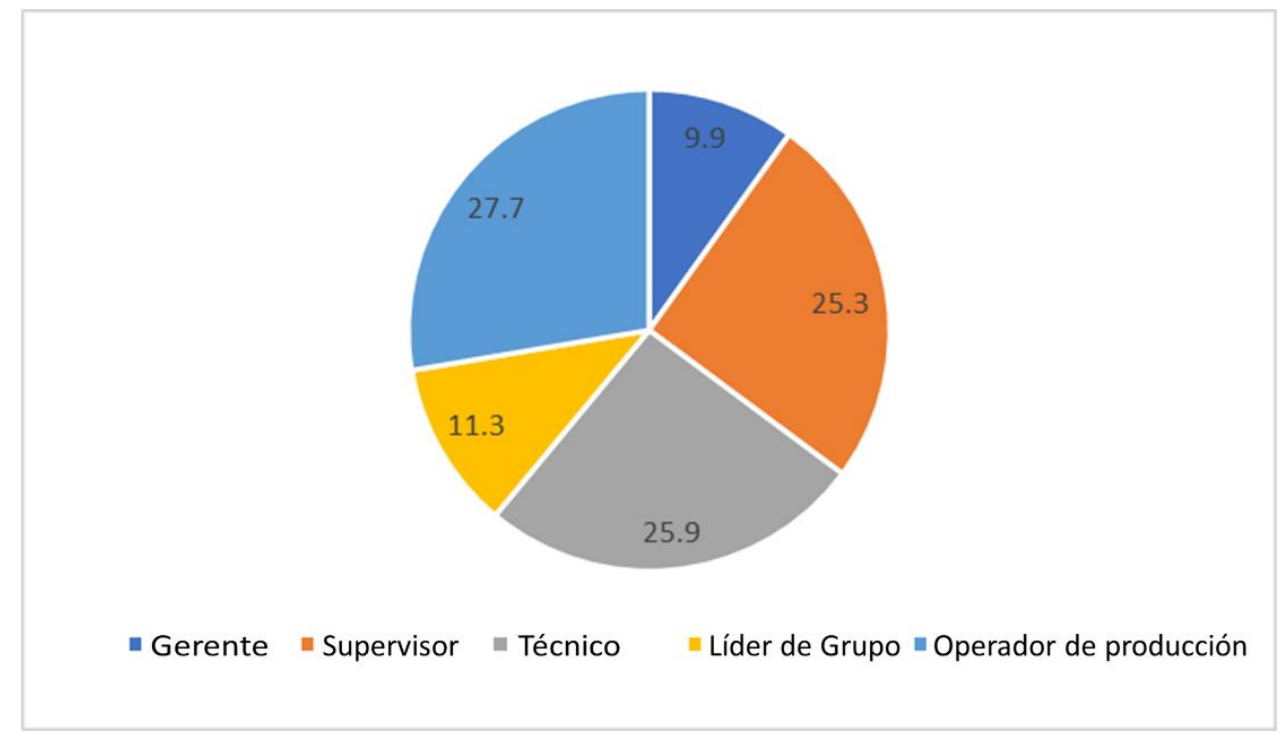

Figura 5. Género de los participantes (\%).

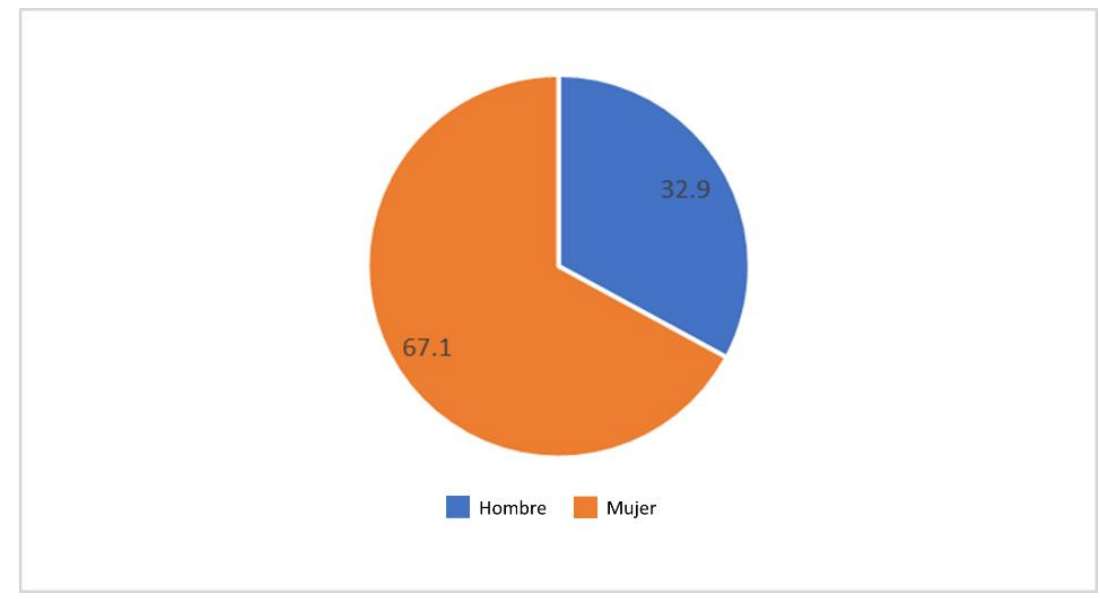


Grados de burnout

La tabla 1 presenta los valores promedio para las dimensiones del burnout.

Tabla 1. Puntuaciones medias de cada dimensión de burnout.

\begin{tabular}{|l|c|}
\hline Dimensión & Valor promedio \\
\hline Agotamiento emocional & 7.85 \\
\hline Cinismo & 7.02 \\
\hline Eficacia profesional & 23.00 \\
\hline
\end{tabular}

Los resultados por grados se presentan en la tabla 2. En consecuencia, los valores medios de la tabla 1 corresponden a un grado medio de Burnout para las tres dimensiones utilizando puntos de corte del 33 y 66 percentil de la muestra dada.

Tabla 2. Grados de burnout.

\begin{tabular}{|l|c|c|c|}
\hline Grado & Agotamiento emocional & Cinismo & Eficacia profesional \\
\hline Bajo & $\leq 5$ & $\leq 4$ & $>30$ \\
\hline Medio & $6-9 \mathbf{( 7 . 8 5 )}$ & $5-9 \mathbf{( 7 . 0 2 )}$ & $19-29(\mathbf{2 3 . 0 0})$ \\
\hline Alto & $>10$ & $>10$ & $\leq 18$ \\
\hline
\end{tabular}

Los resultados muestran que las tres dimensiones del burnout se ubican en un grado medio.

\section{Niveles de burnout}

Los resultados por niveles y sus correspondientes porcentajes en la muestra se presentan en la tabla 3 .

Tabla 3. Niveles de burnout.

\begin{tabular}{|l|c|}
\hline Nivel & Porcentaje \\
\hline Ninguno & 21.97 \\
\hline Bajo & 22.40 \\
\hline Moderado & 20.79 \\
\hline Alto & 17.90 \\
\hline Extremadamente alto & 16.93 \\
\hline
\end{tabular}

\section{Estado civil y burnout}

Con respecto al análisis de varianza, los resultados se muestran en la tabla 4, donde los valores menores al valor $\mathrm{p}<0.05$ son empleados para determinar las diferencias significativas encontradas. Se puede concluir que existe una diferencia significativa en la presencia de burnout según el estado civil de los participantes. 
Tabla 4. Prueba ANOVA con $\alpha=0.05$.

\begin{tabular}{|c|c|c|c|c|c|c|}
\hline & & $\begin{array}{l}\text { Suma de } \\
\text { cuadrados }\end{array}$ & $\begin{array}{c}\text { Grados de } \\
\text { libertad }\end{array}$ & $\begin{array}{c}\text { Media } \\
\text { cuadrada }\end{array}$ & $\mathbf{F}$ & Sig. \\
\hline \multirow{3}{*}{$\begin{array}{l}\text { Agotamiento } \\
\text { emocional }\end{array}$} & Entre grupos & 11.99 & 4 & 2.998 & 4.161 & 0.002 \\
\hline & $\begin{array}{l}\text { Dentro de } \\
\text { grupos }\end{array}$ & 668.505 & 928 & 0.72 & & \\
\hline & Total & 680.495 & 932 & & & \\
\hline \multirow{3}{*}{ Cinismo } & Entre grupos & 7.06 & 4 & 1.765 & 2.673 & 0.031 \\
\hline & $\begin{array}{l}\text { Dentro de } \\
\text { grupos }\end{array}$ & 612.837 & 928 & 0.66 & & \\
\hline & Total & 619.897 & 932 & & & \\
\hline \multirow{3}{*}{$\begin{array}{l}\text { Eficacia } \\
\text { profesional }\end{array}$} & Entre grupos & 12.122 & 4 & 3.03 & 4.69 & 0.001 \\
\hline & $\begin{array}{l}\text { Dentro de } \\
\text { grupos }\end{array}$ & 599.668 & 928 & 0.646 & & \\
\hline & Total & 611.79 & 932 & & & \\
\hline
\end{tabular}

Los resultados de la prueba de Tukey se muestran en la tabla 5. Los resultados por dimensión son:

- Agotamiento emocional: Existe una diferencia en el agotamiento emocional percibido entre los participantes solteros y casados, así como entre los participantes solteros y los que viven en unión libre.

- Cinismo: No hay diferencia significativa en la percepción de cinismo entre los estados civiles estudiados.

- Eficacia profesional: Existe una diferencia en la percepción de eficacia profesional entre los participantes solteros y casados, entre los viudos y los solteros, y entre los viudos y los participantes casados. 
Tabla 5. Prueba de Tukey con $\alpha=0.05$.

\begin{tabular}{|c|c|c|c|c|c|c|c|}
\hline & ble denendi & & Diferencia & Error & Sia & $\begin{array}{r}95 \% \text { In } \\
\text { con }\end{array}$ & $\begin{array}{l}\text { rvalo de } \\
\text { anza }\end{array}$ \\
\hline & & & media (I-J) & estándar & & Límite & Límite \\
\hline Agotamiento & Soltero & Casado & $0.212^{*}$ & 0.063 & 0.007 & 0.04 & 0.38 \\
\hline emocional & & Unión libre & 0.020 & 0.109 & 1.000 & -0.28 & 0.32 \\
\hline & & Viudo & 0.007 & 0.304 & 1.000 & -0.82 & 0.84 \\
\hline & & Divorciado & -0.115 & 0.131 & 0.905 & -0.47 & 0.24 \\
\hline & Unión libre & Soltero & $-0.212^{*}$ & 0.063 & 0.007 & -0.38 & -0.04 \\
\hline & & Unión libre & -0.192 & 0.103 & 0.337 & -0.47 & 0.09 \\
\hline & & Viudo & -0.205 & 0.302 & 0.961 & -1.03 & 0.62 \\
\hline & & Divorciado & -0.327 & 0.127 & 0.075 & -0.67 & 0.02 \\
\hline & Viudo & Soltero & -0.020 & 0.109 & 1.000 & -0.32 & 0.28 \\
\hline & & Casado & 0.192 & 0.103 & 0.337 & -0.09 & 0.47 \\
\hline & & Viudo & -0.013 & 0.315 & 1.000 & -0.87 & 0.85 \\
\hline & & Divorciado & -0.135 & 0.155 & 0.906 & -0.56 & 0.29 \\
\hline & Divorciado & Soltero & -0.007 & 0.304 & 1.000 & -0.84 & 0.82 \\
\hline & & Casado & 0.205 & 0.302 & 0.961 & -0.62 & 1.03 \\
\hline & & Unión libre & 0.013 & 0.315 & 1.000 & -0.85 & 0.87 \\
\hline & & Divorciado & -0.122 & 0.324 & 0.996 & -1.01 & 0.76 \\
\hline & Casado & Soltero & 0.115 & 0.131 & 0.905 & -0.24 & 0.47 \\
\hline & & Casado & 0.327 & 0.127 & 0.075 & -0.02 & 0.67 \\
\hline & & Unión libre & 0.135 & 0.155 & 0.906 & -0.29 & 0.56 \\
\hline & & Viudo & 0.122 & 0.324 & 0.996 & -0.76 & 1.01 \\
\hline Cinismo & Soltero & Casado & 0.103 & 0.060 & 0.432 & -0.06 & 0.27 \\
\hline & & Unión libre & 0.252 & 0.104 & 0.110 & -0.03 & 0.54 \\
\hline & & Viudo & 0.521 & 0.291 & 0.380 & -0.28 & 1.32 \\
\hline & & Divorciado & 0.246 & 0.126 & 0.290 & -0.10 & 0.59 \\
\hline & Unión libre & Soltero & -0.103 & 0.060 & 0.432 & -0.27 & 0.06 \\
\hline & & Unión libre & 0.150 & 0.099 & 0.553 & -0.12 & 0.42 \\
\hline & & Viudo & 0.419 & 0.290 & 0.598 & -0.37 & 1.21 \\
\hline & & Divorciado & 0.143 & 0.121 & 0.763 & -0.19 & 0.48 \\
\hline & Viudo & Soltero & -0.252 & 0.104 & 0.110 & -0.54 & 0.03 \\
\hline & & Casado & -0.150 & 0.099 & 0.553 & -0.42 & 0.12 \\
\hline & & Viudo & 0.269 & 0.302 & 0.900 & -0.56 & 1.09 \\
\hline & & Divorciado & -0.006 & 0.148 & 1.000 & -0.41 & 0.40 \\
\hline & Divorciado & Soltero & -0.521 & 0.291 & 0.380 & -1.32 & 0.28 \\
\hline & & Casado & -0.419 & 0.290 & 0.598 & -1.21 & 0.37 \\
\hline & & Unión libre & -0.269 & 0.302 & 0.900 & -1.09 & 0.56 \\
\hline & & Divorciado & -0.276 & 0.310 & 0.901 & -1.12 & 0.57 \\
\hline & Casado & Soltero & -0.246 & 0.126 & 0.290 & -0.59 & 0.10 \\
\hline & & Casado & -0.143 & 0.121 & 0.763 & -0.48 & 0.19 \\
\hline & & Unión libre & 0.006 & 0.148 & 1.000 & -0.40 & 0.41 \\
\hline
\end{tabular}




\begin{tabular}{|c|c|c|c|c|c|c|c|}
\hline & & Viudo & 0.276 & 0.310 & 0.901 & -0.57 & 1.12 \\
\hline \multirow{20}{*}{$\begin{array}{l}\text { Eficacia } \\
\text { profesional }\end{array}$} & \multirow[t]{4}{*}{ Soltero } & Casado & 0.001 & 0.060 & 1.000 & -0.16 & 0.16 \\
\hline & & Unión libre & $-0.355^{*}$ & 0.103 & 0.005 & -0.64 & -0.07 \\
\hline & & Viudo & -0.310 & 0.288 & 0.818 & -1.10 & 0.48 \\
\hline & & Divorciado & -0.265 & 0.124 & 0.210 & -0.60 & 0.08 \\
\hline & \multirow[t]{4}{*}{ Unión libre } & Soltero & -0.001 & 0.060 & 1.000 & -0.16 & 0.16 \\
\hline & & Unión libre & $-0.357^{*}$ & 0.098 & 0.003 & -0.62 & -0.09 \\
\hline & & Viudo & -0.312 & 0.286 & 0.812 & -1.09 & 0.47 \\
\hline & & Divorciado & -0.266 & 0.120 & 0.175 & -0.59 & 0.06 \\
\hline & \multirow[t]{4}{*}{ Viudo } & Soltero & $0.355^{*}$ & 0.103 & 0.005 & 0.07 & 0.64 \\
\hline & & Casado & $0.357^{*}$ & 0.098 & 0.003 & 0.09 & 0.62 \\
\hline & & Viudo & 0.045 & 0.298 & 1.000 & -0.77 & 0.86 \\
\hline & & Divorciado & 0.091 & 0.147 & 0.972 & -0.31 & 0.49 \\
\hline & \multirow[t]{4}{*}{ Divorciado } & Soltero & 0.310 & 0.288 & 0.818 & -0.48 & 1.10 \\
\hline & & Casado & 0.312 & 0.286 & 0.812 & -0.47 & 1.09 \\
\hline & & Unión libre & -0.045 & 0.298 & 1.000 & -0.86 & 0.77 \\
\hline & & Divorciado & 0.046 & 0.307 & 1.000 & -0.79 & 0.88 \\
\hline & \multirow[t]{4}{*}{ Casado } & Soltero & 0.265 & 0.124 & 0.210 & -0.08 & 0.60 \\
\hline & & Casado & 0.266 & 0.120 & 0.175 & -0.06 & 0.59 \\
\hline & & Unión libre & -0.091 & 0.147 & 0.972 & -0.49 & 0.31 \\
\hline & & Viudo & -0.046 & 0.307 & 1.000 & -0.88 & 0.79 \\
\hline
\end{tabular}

Nota. ${ }^{*}$ La diferencia media es significativa al nivel de 0.05 .

\section{Conclusiones}

Se concluye que la presencia de burnout en la muestra presenta un grado medio en todas las dimensiones. En referencia al burnout por niveles, el 75\% de la muestra presenta algún nivel de burnout, y más del 30\% lo presenta en niveles altos y extremadamente altos. Por lo tanto, los gerentes medios y superiores de la industria maquiladora están experimentando los efectos del burnout que afectan negativamente su salud, productividad y desempeño (Armenta-Hernández et al., 2021). Además, este estudio encontró algún grado y nivel de agotamiento emocional en todos los estados civiles estudiados. Sin embargo, los análisis estadísticos mostraron diferencias significativas solo en la dimensión de agotamiento emocional, en la cual estar casado es el estado civil que presenta esta diferencia respecto al resto de estados civiles. Los resultados obtenidos en esta investigación difieren de los encontrados por Facal-Fondo (2012), donde se encontraron relaciones no significativas entre el estado civil y el agotamiento emocional. Por el contrario, coinciden con aquellos obtenidos por Ramírez (2017) y Picasso-Pozo et al. (2012), quienes encontraron que el estado civil está directamente relacionado al burnout. 


\section{Agradecimientos}

Los autores agradecen a las empresas participantes sus invaluables contribuciones y participación. Asimismo, agradecemos a la Universidad Autónoma de Ciudad Juárez (UACJ) y al Consejo Nacional de Ciencia y Tecnología (CONACYT) por el apoyo financiero otorgado a través del proyecto CONACYT-INS (FRONTERAS CIENCIA) 2016-01-2433. 


\section{Referencias}

Aguirre, N. H., Medellín, J., Vázquez, L., Gutiérrez, G., \& Fernández, M. C. (2014). Síndrome de quemarse por el trabajo y su relación con el puesto de trabajo en empleados de la industria maquiladora. Revista PsicologiaCientifica.com, 16(1). http://www.psicologiacientifica.com/sindromequemarse-trabajo-relacion-puesto-trabajoempleados

Armenta-Hernández, O., Maldonado-Macías, A., Camacho-Alamilla, M. D. R., Serrano-Rosa, M. Á., Baez-Lopez, Y. A., \& Balderrama-Armendariz, C. O. (2021). The relationship between the burnout syndrome dimensions and body mass index as a moderator variable on obese managers in the mexican maquiladora industry. Frontiers in Psychology, 12, 127. https://doi.org/10.3389/fpsyg.2021.540426

Ávila Toscano, J. H., Gómez Hernández, L. T., \& Montiel Salgado, M. M. (2010). Características demográficas y laborales asociadas al síndrome de burnout en profesionales de la salud. Pensamiento Psicológico, $\quad 8(15), \quad 39-52$. https://revistas.javerianacali.edu.co/index.php/pensamientopsicologico/article/view/142

Bautista, O., Tombé, P., \& Victoria, M. (2013). Síndrome de burnout y su relación con el estado de salud mental en un grupo de trabajadores de seguridad privada. Revista Colombiana de $\begin{array}{lll}\text { Salud } & \text { Ocupacional, 20-24. }\end{array}$ https://revistas.unilibre.edu.co/index.php/rc salud ocupa/article/view/4872

Facal-Fondo, T. (2012). Prevalencia del síndrome de burnout en trabajadores sociales de los

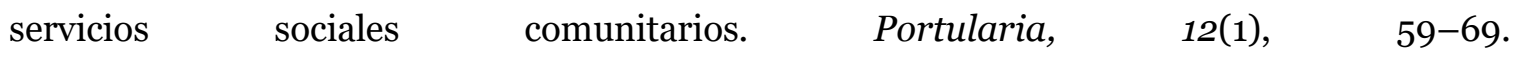
https://doi.org/10.5218/prts.2012.0034

González, A. \& D. (2019). Estrés laboral, ladrón de la salud y la productividad empresarial. http://lanotaeconomica.com.co/pildoras-ejecutivas/estres-laboral-ladron-de-la-salud-y-laproductividad-empresarial.html

González-Ramírez, M. T., \& Landero-Hernández, R. (2008). Confirmación de un modelo explicativo del estrés y de los síntomas psicosomáticos mediante ecuaciones estructurales. $\begin{array}{lllll}\text { Revista Panamericana de } & \text { Salud }\end{array}$ https://iris.paho.org/handle/10665.2/7746

Guerrero, E. \& Vicente, F. (2001). Síndrome de burnout o desgaste profesional y estrés en el profesorado. Servicio de publicaciones de la Universidad de Extremadura.

Instituto Mexicano del Seguro Social (2018). Estrés Laboral. http://www.imss.gob.mx/salud-enlínea/estres-laboral

Izquierdo-Botica, F. (2019). Estrés laboral: No dejes que entre en tu vida. Fundación Española del Corazón. https://fundaciondelcorazon.com/corazon-facil/blog-impulso-vital/2499estres-laboral-no-dejes-que-entre-vida.html

Moreno-Jiménez, B., Rodríguez-Carvajal, R., \& Escobar Redonda, E. (2001). La evaluación del burnout profesional. Factorialización del MBI-GS. Un análisis preliminar. Ansiedad y Estrés, $7(1), \quad 69-78 . \quad$ http://www.integraorg.com/wpcontent/docs/La\%20Evaluacion\%20del\%20Burnout\%20Profesional.pdf

Peralta-Ayala C. \& Moya-Silva T. J. (2017). Síndrome de burnout en estudiantes de odontología. Revista Científica Dominio de las Ciencias, 3(1), 432-442. http://dx.doi.org/10.23857/dom.cien.pocaip.2017.3.1.432-442

Picasso-Pozo, M. Á., Huillca-Castillo, N., Ávalos-Márquez, J. C., Omori-Mitumori, E., GallardoSchultz, A., \& Salas-Chávez, M. (2012). Síndrome de burnout en estudiantes de odontología 
de una universidad $\quad$ peruana. $\quad$ Kiru, $\quad 9(1), \quad 51-58$. https://repositorio.usmp.edu.pe/handle/20.500.12727/1870

Quezada-Berumen, L. C., \& González-Ramírez, M. T. (2011). Relación entre depresión, estrés y estresores en adolescentes gestantes bajo distintas condiciones de estado civil. Summa Psicológica, 8(2), 31-38. https://doi.org/10.18774/448x.2011.8.85

Ramírez, M. R. (2017). Prevalencia del síndrome de burnout y la asociación con variables sociodemográficas y laborales en una provincia de Ecuador. International Journal of Developmental and Educational Psychology, 4(1), 241-252. https://doi.org/10.17060/ijodaep.2017.n1.v4.1053

Sandín, B. (2003). El estrés: Un análisis basado en el papel de los factores sociales. International Journal of clinical and health psychology, 3(1), 141-157. https://www.redalyc.org/pdf/337/33730109.pdf 